\title{
Setting of the ATLAS Jet Energy Scale
}

\author{
Michele Petteni ${ }^{* \dagger}$ \\ Simon Fraser University \\ E-mail: mpetteni@sfu.ca
}

The setting of the jet energy scale and its uncertainty in the ATLAS detector is presented. After a brief introduction of the ATLAS calorimeter and the jet reconstruction algorithms used in ATLAS, the determination of the jet energy scale is described. Emphasis is given to the differing approaches adopted by ATLAS. The current approach used for analysis is highlighted and results from early collision data are presented. The current jet energy scale for inclusive jets with a transverse momentum of $100 \mathrm{GeV}$, is determined with an uncertainty of 6-7\%.

Kruger 2010: Workshop on Discovery Physics at the LHC

December 5 - 10, 2010

Kruger National Park, Mpumalanga, South Africa

* Speaker.

${ }^{\dagger}$ On Behalf of the ATLAS Collaboration 


\section{Introduction}

The determination of the jet energy scale is a central part of any physics analysis involving jets in final states. The jet energy scale uncertainty is the dominant experimental systematic uncertainty in a number of physics analysis, such as the top mass measurement, and the measurement of the di-jet cross-section.

The jet energy scale allows experiments to relate the energy and transverse momentum as measured in the calorimeter to the hadron level. Thus aiming to correct for a variety of instrumental and detector effects, including the intrinsically different response of the calorimeter to electromagnetic and hadronic deposits, reconstruction effects, dead material, noise and pile-up, etc. In ATLAS the jet energy scale is determined from Monte Carlo.

The ATLAS detector covers $|\eta|<4.9$ around the collision point with layers of tracking detectors (covering $|\eta|<2.5$ ), calorimeters and muon chambers [1]. The calorimeter is composed of a high granularity liquid-argon electromagnetic sampling calorimeters (LAr), divided into three cryostats, which cover the pseudo-rapidity range $|\eta|<3.2$. The hadronic calorimetry in the range $|\eta|<1.7$ is provided by a sampling calorimeter made of steel and scintillating tiles (Tile). Enclosed in the end-cap cryostats $(|\eta|>1.5)$, LAr technology is also used for the hadronic calorimeters (HEC), matching the outer $|\eta|$ limits of the end-cap electromagnetic calorimeters. The LAr forward calorimeters (FCal) is a liquid argon and tungsten/copper detector, and provides both electromagnetic and hadronic energy measurements out to $|\eta|<4.9$

\section{Jet Reconstruction in ATLAS}

ATLAS uses a variety of jet reconstruction algorithms of which the anti- $k_{t}$ [2] is the most commonly used. The anti- $k_{t}$ algorithm is a sequential recombination style algorithm which can take as input any 4-vector quantity. In ATLAS, resolution parameters $\mathrm{R}=0.4$ and $\mathrm{R}=0.6$ are used. As inputs to the jet finder two clustering algorithms are adopted. For truth jets all stable particles excluding muons and neutrinos are used. The clusters input to the jet finding are treated as if arising from massless particles.

The topological clustering algorithm starts with seed cells required to have $|E|>4 \sigma_{\text {rms noise }}$. Nearest neighbours with $|E|>2 \sigma_{\text {rms noise }}$ are added to the proto-cluster. If no neighbouring cells are found satisfying this requirement the proto-cluster is rejected. Neighbours are added until there are no more cells satisfying the neighbour requirement, at which point all the subsequent neighbouring cells are added as a "guard ring". A split-merge algorithm is applied to all final clusters.

Due to the iterative nature of the algorithm, the topological clusters formed have no fixed size and no geometrical relation to the layout of the calorimeter. In order to provide an alternative fixed size signal definition, topological-towers are built by segmenting the topological clusters into a fixed size $\Delta \eta \times \Delta \phi=0.1 \times 0.1$ grid. The physical size of the towers varies with $\eta$ and longitudinal segmentation, but is generally the same size as this grid.

\section{Determination of the Jet Energy Scale}

The baseline for the jet energy scale determination is the electromagnetic (EM) scale, as determined from the response of electrons in the LAr and Tile calorimeters in test beam data. Subsequent 

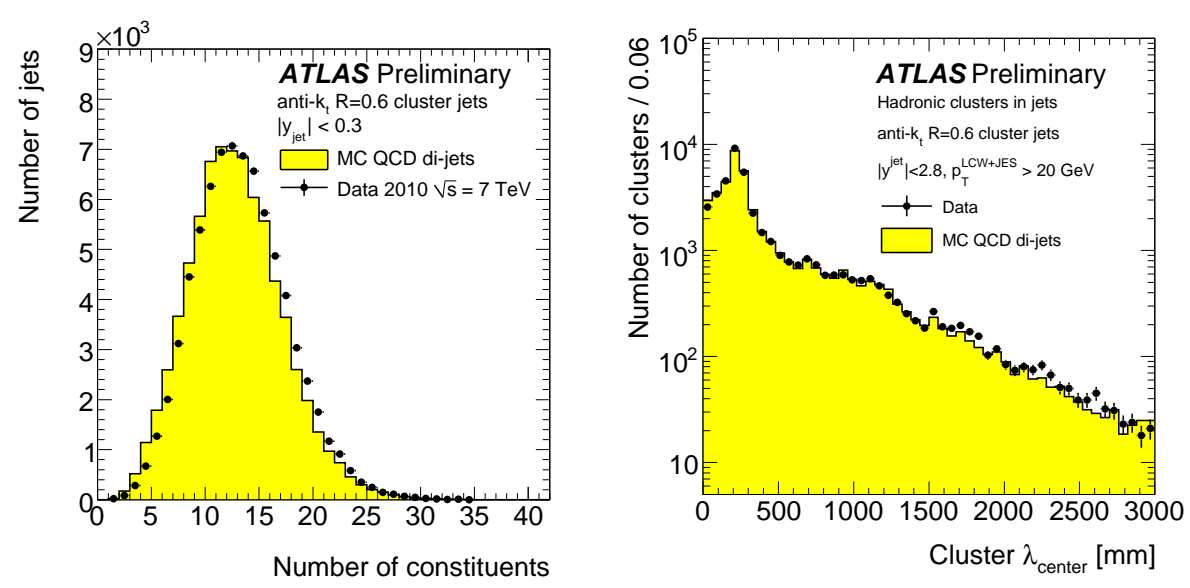

Figure 1: (a) Number of constituents in jets built with and topological clusters, and (b) distribution, $\lambda_{\text {center }}$ of the longitudinal centre of hadronic clusters in data (points) and Monte Carlo simulation (histograms).

to this initial scale, jets can be corrected on two levels, via the properties of the clusters or cells and using the jet kinematics. The jet level corrections can be derived independently of whether the constituent/cell level corrections are implemented. The constituent level corrections, however, also require an overall jet energy scale correction to be derived. Currently ATLAS uses a jet energy scale calculated from di-jet Monte Carlo simulation.

\subsection{Constituent Level Corrections}

ATLAS has adopted two methods for constituent based corrections: Global Cell Weighting (GCW) and Local Cluster Weighting (LCW) [3]. Both methods rely on the differences in shower profiles between electromagnetic and hadronic signals.

Local Cluster Weighting is applied to the constituents before jet reconstruction. LCW differentiates hadronic and electromagnetic clusters based on shower depth, cell-energy density, cluster energy and $\eta$. Based on these variables the clusters can be weighted to correct for the hadronic response, dead material and out-of-cluster deposits using templates from the simulation of single pions.

Fig 1 shows the Monte Carlo and data comparison for some of the inputs for the LCW. The cluster shapes match well although there is a shift of around one cluster for the number of constituents. There is good closure for most of the calorimeter except for the transition region between the central and end-cap cryostats, where discrepancies of up to $10 \%$ are observed.

Global Cell Weighting is applied to jets after reconstruction. Hadronic and EM energy deposits can be distinguished via the lower energy volume density, E/V, of the hadronic showers. Cell weights binned in $\mathrm{E} / \mathrm{V}, \eta$ and calorimeter layer are calculated by minimising the energy resolution of reconstructed jets in di-jet Monte Carlo. Hence by applying these weights to the cells of jets in data, some of the difference between hadronic and EM response can be corrected for.

Fig 2 shows the E/V distribution for cells in a hadronic and EM calorimeter layer. There is good agreement between data and simulation in the hadronic layer, whereas the EM layer shows a slight deficit in data of cells with high energy density when compared to Monte Carlo. 

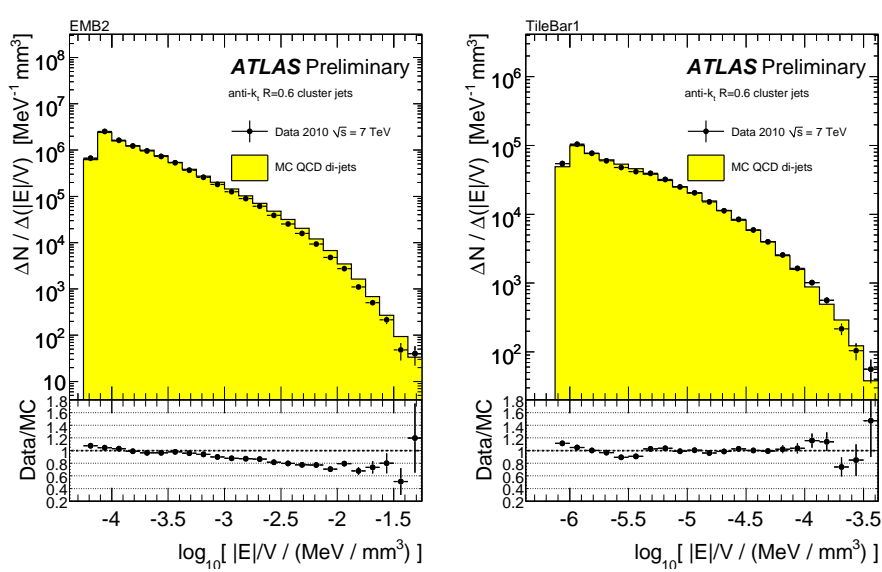

Figure 2: Cell energy density distributions used in the GCW jet calibration scheme in data (points) and Monte Carlo simulation (histograms) for cells in (a) the second layer of the barrel electromagnetic calorimeter and (b) in the second layer of the barrel hadronic Tile calorimeter. Monte Carlo simulation distributions are normalized to the number of cells in data distributions.

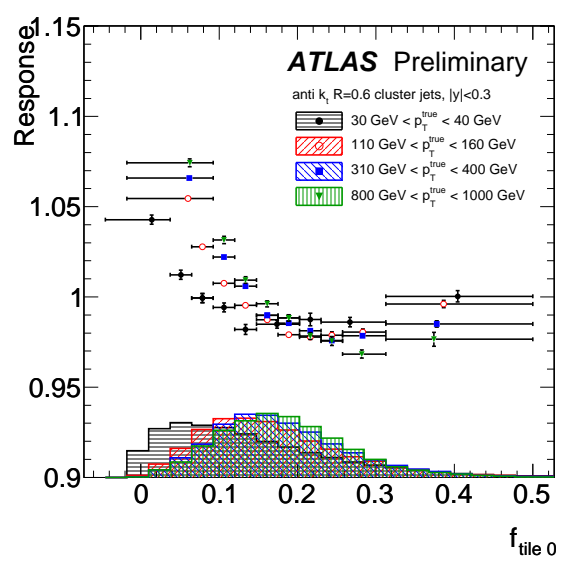

Figure 3: Jet energy response as a function of the fraction of the electromagnetic-scale jet energy deposited in the first layer of the Tile calorimeter.

\subsection{Jet Level Corrections}

The purpose of the jet energy scale (JES) is to correct the energy of jets, either at the EM-scale or after constituent based calibrations, back to particle level. The current ATLAS method derives the JES as a function of $\eta$ and reconstructed jet $p_{T}$ using the truth level information in di-jet Monte Carlo as a reference [4].

A further technique, Global Sequential Calibration (GSC), can also be applied using jet level quantities. The aim of GSC is not to correct the scale itself but to reduce fluctuations in the jet response in order to reduce the overall jet energy resolution. It uses the same technique as the main JES derivation but parametrises the jet response in a sequence of secondary variables. As the correction is applied after jet calibration, no additional jet energy scale is required. 


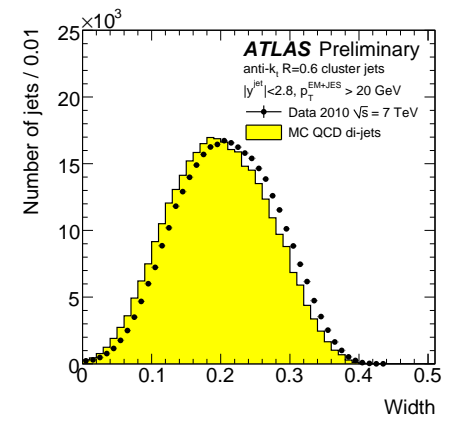

(a)

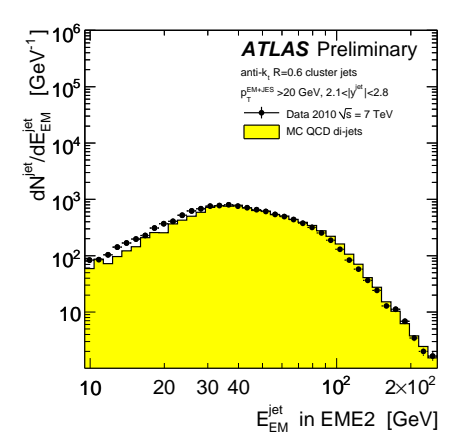

(b)

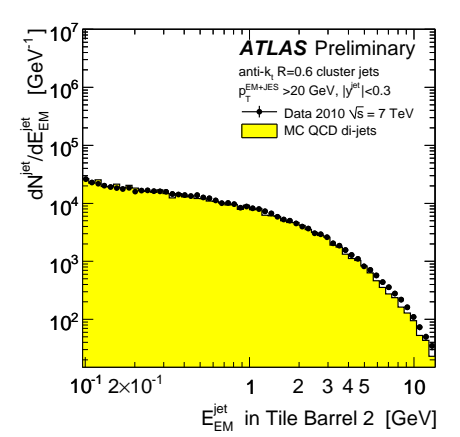

(c)

Figure 4: Distribution in data (points) and Monte Carlo simulation (histograms) for a) jet width distribution, b) energy deposited by jets second layer of the end-cap electromagnetic calorimeter and c) that deposited in the second layer of the hadronic calorimeter in the central region.

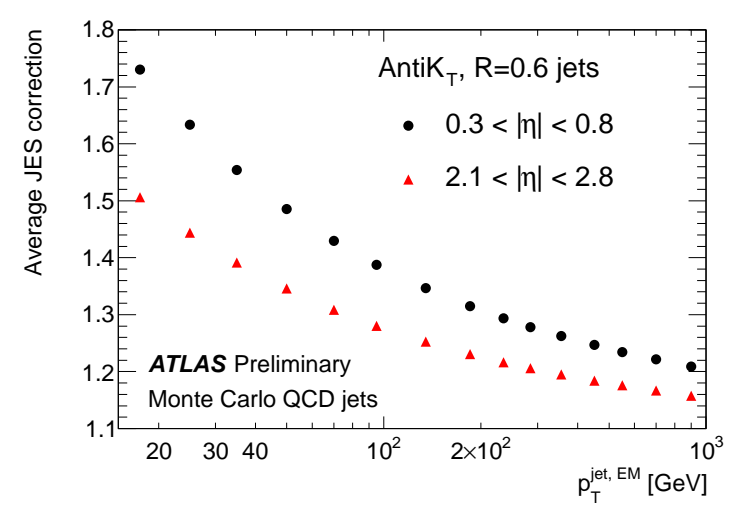

Figure 5: Average jet energy scale correction as a function of jet transverse momentum at the electromagnetic scale $p_{T}^{\text {jet,EM }}$ for jets in the central barrel (black circles) and endcap (red triangles) regions

Fig 3 show the dependence of the jet response on the fractional jet energy in the Tile 0 layer, one the variables used in the GSC method. It is clear that in parametrising the jet response as a function of this variable the overall jet response will have a smaller spread, and hence a more accurate determination.

Fig 4 show the data and Monte Carlo distributions of the inputs used in GSC. It can be seen that the width seen in data is not properly described by the Monte Carlo. Further tuning of the Monte Carlo is needed for the width to be described properly.

\section{Jet Energy Scale Derivation}

Fig 5 shows the Jet Energy Scale correction for anti- $k_{t}, \mathrm{R}=0.6 \mathrm{EM}$ scale jets as derived in PYTHIA di-jet Monte Carlo. The JES uncertainty is displayed in Fig 6 a). This uncertainty is calculated by re-deriving the jet response after varying the Monte Carlo under different detector configurations, dead material budgets, noise in the calorimeter and via using an in-situ $\eta$ inter- 

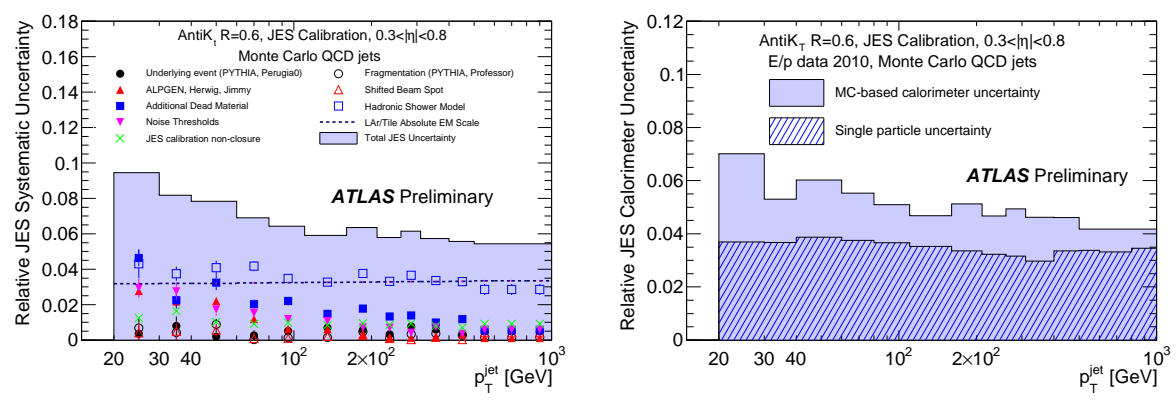

Figure 6: Relative jet energy scale systematic uncertainty as a function of $p_{T}^{\text {jet }}$ for jets in the central calorimeter barrel region (a) The total uncertainty is shown as the solid light blue area. The individual sources are also shown, with statistical errors if applicable, (b) due to the calorimeter measurement estimated from single particle analysis (hatched) and from Monte Carlo studies (solid).

calibration to extend the uncertainty to the forward region. In addition different models for the hadronic shower, fragmentation and underlying event and an overall generator dependence are used. The dominant contributions to the uncertainty are the dead material description and the hadronic shower modelling, each of which contributes $5 \%$ at low $p_{T}$. At a slightly lower level of $3 \%$ are contributions from the noise description, the absolute EM-scale determination from test beam measurements and from the $\eta$ inter-calibration between the different cryostat regions.

The jet energy scale uncertainty is smaller the $7 \%$ for jets with a $p_{T}>100 \mathrm{GeV}$. Due to the uncertainty of the modelling of the Monte Carlo in the forward region $(\eta>3.2)$, the jet energy scale uncertainty has been assessed to $|\eta|<4.5$ using di-jet balance measurements derived from data [6]. The current jet energy scale does not include flavour dependence or corrections for the jet isolation. The effect of in-time pile-up was taken into account directly from data, using measurements of tower energy density as a function of the number of additional multiple interactions.

\subsection{Closure Tests}

The calorimeter uncertainty is verified using the single hadron jet response as measured from minimum bias data [5]. The momentum computed from isolated tracks is used to calculate the jet response, E/p, for single particles. This is then extrapolated to a jet energy scale uncertainty via Monte Carlo pseudo-experiments. The comparison of the two calorimeter uncertainties is shown in Fig 6 b), where the reduction in the uncertainty measurement from the in-situ method is evident.

Fig 7 displays the Data-MC double ratio of jet energy, as a function of jet $p_{T}$ for the three additional calibration methods. As one can see there is good closure between the Monte Carlo and data and also between the different methods. The overall disagreement in the input distributions has a $2 \%$ impact on the jet energy scale. These will improve with a better tuning of the Monte Carlo.

\section{Conclusions}

The first determination of the jet energy scale with the ATLAS detector has been performed. The jet energy scale is derived from Monte Carlo and applied to EM calibrated jets. This method 


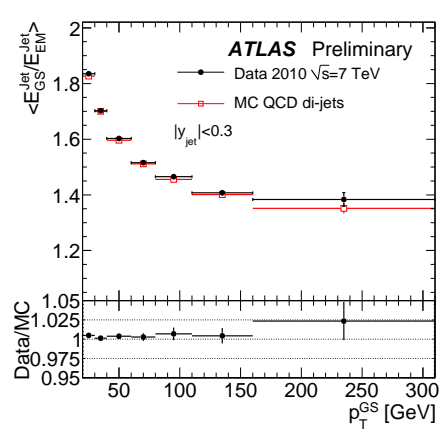

(a)

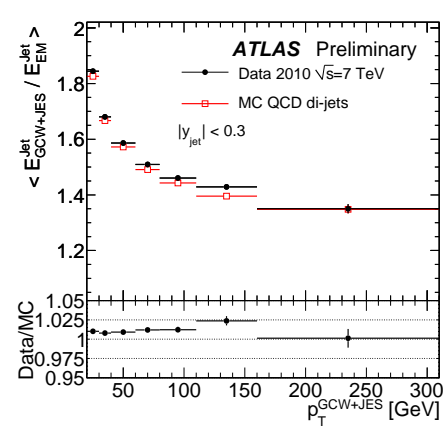

(b)

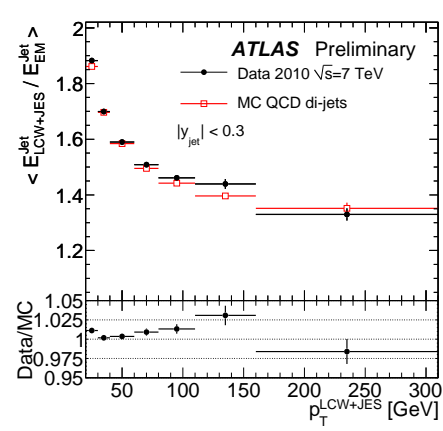

(c)

Figure 7: Mean calibrated jet energy over uncalibrated jet energy as a function of calibrated jet $p_{T}$ for jets constructed from topological clusters calibrated with (a) the global sequential, (b) the global cell energydensity weighting, and (c) local cluster weighting calibration schemes.

gives a precision of $7 \%$ for jets with $p_{T}>100 \mathrm{GeV}$. In-situ methods, used as a cross-check, have already demonstrated a better precision. The switch to using calibrated cell inputs is also expected to give a smaller jet energy resolution. The precision of the measurement will increase with a better Monte Carlo modelling and with a more accurate EM calibration from $\mathrm{Z}$ peak measurements. The use of in-situ methods, such as $\gamma+$ jets, multi-jet balance and track jets, is expected to not only to provide a valuable cross-check of the jet energy scale but also to improve the uncertainty especially for high- $p_{t}$ jets. A significant reduction of the systematic uncertainty is to be expected in the near future [7].

\section{References}

[1] The ATLAS Collaboration, The ATLAS Experiment at the CERN Large Hadron Collider JINST 3, S08003, (2008)

[2] M. Cacciari, G. P. Salam and G. Soyez, The anti- $k_{t}$ jet clustering algorithm, JHEP 04 (2008) 063 [hep-ph/0802.1189]

[3] The ATLAS Collaboration, Properties of Jets and Inputs to Jet Reconstruction and Calibration with the ATLAS Detector Using Proton-Proton Collisions at $\sqrt{s}=7 \mathrm{TeV}$, ATL-CONF-2010-053 (2010)

[4] The ATLAS Collaboration, Jet energy scale and its systematic uncertainty in ATLAS for jets produced in proton-proton collisions at sqrts $=7 \mathrm{TeV}, A T L-C O N F-2010-058$ (2010)

[5] The ATLAS Collaboration, Jet energy resolution and selection efficiency relative to track jets from in-situ techniques with the ATLAS Detector Using Proton-Proton Collisions at a Center of Mass Energy sqrts $=7 \mathrm{TeV}$, ATL-CONF-2010-054 (2010)

[6] The ATLAS Collaboration, In-situ pseudo-rapidity inter-calibration to evaluate jet energy scale uncertainty and calorimeter performance in the forward region, ATL-CONF-2010-055 (2010)

[7] The ATLAS Collaboration, Jet energy scale and its systematic uncertainty in proton-proton collisions at sqrt(s)=7 TeV in ATLAS 2010 data, ATL-CONF-2011-032 (2011) 